astérisques. Il y existe donc au moins une ligne qui contient $\geqslant k-2$ astérisques, car, en eas contraire, le nombre des astérisques serait

$$
\leqslant(k-3)(2 k-3)=2 k^{2}-9 k+9,
$$

ce qui est impossible.

La conclusion suit de la remarque 1 du NI.

Par raison de symétrie, on peut remplacer $k$ par $n$ et $n$ par $k$.

Instytut Matematyezny Polskiej Akademii Nank.

\section{Concerning the Homological Structure of the Functional Space $S_{m}^{X}$.}

By

\section{Karol Borsuk (Warszawa).}

1. Functional space. Let $X$ be a compact space and $Y$ a metric space. We denote the set of all continuous mappings of $X$ in $Y$ by $Y^{X}$ and we define a metric in $Y^{X}$ by setting

$$
\left.\varrho(\varphi, \psi)=\operatorname{Sup}_{x \in X} Q(q(x)), \psi(x)\right) \text { for every } \varphi, \psi \in Y^{X} .
$$

The functional spaces $Y^{X}$ offer scope for ample investigation especially when $Y$ is the Euclidean $m$-dimensional sphere $S_{m}$. In particular the problem of what properties of $X$ imply the connectedness of $S_{m}^{X}$ is completely resolved for the case $\operatorname{dim} X \leqslant m$, by the celebrated theorem of $H$. $\mathrm{Hopf}^{1}$ ). The relations between the properties of $\boldsymbol{X}$ and the other properties of $\mathcal{S}_{m}^{\boldsymbol{X}}$ have been less fully investigated. The purpose of this paper is to indicate a simple relation between some homological properties of $X$ and of $S_{m}^{X}$.

2. $\varepsilon$-chains in a metric space. Let $M$ be a metric space. By an $\varepsilon$-simplex of $M$ we understand a finite subset of $M$ with diameter $<\varepsilon$. In the known manner we introduce the notion of an oriented $\varepsilon$-simplex, of an $\varepsilon$-chain with arbitrarily given coefficients and especially of an $\varepsilon$-cycle. If the group of coefficients is the group of rational numbers $\Re$ then the chains will be said to be rational. The boundary of a chain $x$ will be denoted by $\partial x$. Let us point ont

1) H. Hopf, Die Klassen der Abbildungen der n-dimensionalen Polyeder auf die n-dimensionale Sphäre, Comm. Math. Helv. 5 (1933), p. 39-54 (for polytopes), and H. Freudenthal, Bettische Gruppe mod I und Hopjsche Gruppe, Compositio Math. 4 (1937), p. 235-238 (for compact spaces). See also W. Hurewicz and H. Wallmann, Dimension Theory, Princeton 1941, p. 147. 
that by the boundary of a (0-dimensional simplex. (a) we understand the number 1 . Consequently a 0 -dimensional chain is a cycle if and only if the sum of its coefficients vanishes.

Two $\varepsilon$-cycles $\gamma$ and $\gamma^{\prime}$ of $M$ (with coefficients belonging to an arbitrarily given abelian group $\mathfrak{A}$ ) are called $\eta$-homologous in $M$ (notation: $\gamma \tilde{\eta} \gamma^{\prime}$ in $M$ ) if there exists an $\eta$-chain $x$ of $M$ (with the coefficients belonging to $\mathfrak{A}$ ) such that $\partial x=\gamma-\gamma^{\prime}$.

An $\varepsilon$-simplex $\left(a_{0}, a_{1}, \ldots, a_{n}\right)$ is said to be degenerated, if not all vertices $a_{0}, a_{1}, \ldots, a_{n}$ are different. We assume that the degenerated simplexes can be added or cancelled in a chain without changing it. The boundary of a degenerate simplex vanishes.

3. Cartesian products. Let $\zeta$ be an ordering relation defined in the set $M$, i. e. a relation defined for each pair of different points $x, y \in M$, asymmetric and transitive. Evidently each $k$-dimensional $\varepsilon$-chain $x$ of $\boldsymbol{M}$ can be uniquely represented by the sum

$$
\varkappa=\sum_{\nu=1}^{n} \alpha_{\nu}\left(a_{\nu, 0}, a_{\nu, 1}, \ldots, a_{\nu, k}\right)
$$

where $a_{\nu} \neq 0$ and $a_{\nu, i} \zeta a_{\nu, j}$ for every $\nu=1,2, \ldots, n$ and $i<j$. The representation (1) of $x$ will be said to be consistent with the order $\zeta$.

Let $M$ and $M^{\prime}$ be two metric spaces and let $M \times M^{\prime}$ denote their Cartesian product, i. e. the metric space whose points are ordered pairs $x \times x^{\prime}$ with $x \in M$ and $x^{\prime} \in M^{\prime}$ and such that the metric is defined by the formula

$$
\varrho\left(x \times x^{\prime}, y \times y^{\prime}\right)=\sqrt{\varrho(x, y)^{2}+\varrho\left(x^{\prime}, y^{\prime}\right)^{2}} .
$$

Let $\zeta$ denote an ordering relation in $M$ and $\dot{\gamma}$ an ordering relation in $\boldsymbol{M}^{\prime}$. Let us assign to each pair of non-degenerate simplexes $\Delta=\left(a_{0}, a_{1}, \ldots, a_{k}\right)$ of $M$ and $\Delta^{\prime}=\left(a_{0}^{\prime}, a_{1}^{\prime}, \ldots, a_{k^{\prime}}^{\prime}\right)$ of $M^{\prime}$ in which the vertices are ordered consistently with the relations $\underset{\zeta}{\zeta}$ and $\dot{\vec{\zeta}}$, the chain $\Delta \times \Delta^{\prime}$ (with integral coefficients) given by the formula:

$$
\begin{gathered}
\Delta \times \Delta^{\prime}=\left(a_{0}, a_{1}, \ldots, a_{k}\right) \times\left(a_{0}^{\prime}, a_{1}^{\prime}, \ldots, a_{k^{\prime}}^{\prime}\right)= \\
=\Sigma \pm\left(a_{t_{0}} \times a_{t_{0}^{\prime}}^{\prime}, a_{i_{1}} \times a_{t_{1}^{\prime}}^{\prime}, \ldots, a_{i_{k+k^{\prime}}} \times a_{i_{k+k^{\prime}}^{\prime}}^{\prime}\right),
\end{gathered}
$$

the sum being extended over all non-decreasing sequences of indices $i_{0}, i_{1}, \ldots, i_{k+k^{\prime}}$ and $i_{0}^{\prime}, i_{1}^{\prime}, \ldots, i_{k+k^{\prime}}^{\prime}$ such that

$$
\left.0 \leqslant i_{0}+i_{0}^{\prime}<i_{1}+i_{1}^{\prime}<\ldots<i_{k+k^{\prime}}+i_{k+k^{\prime}}^{\prime} \leqslant k+k^{\prime 2}\right) .
$$

2) See H. Freudenthal, Eine Simplizialzerlegung des Oartesischen Produktes zweier Simplexe, Fund. Math. 29 (1937), p. 139.
We see at once that if $\Delta$ is an $\varepsilon$-simplex and $\Delta^{\prime}$ an $\varepsilon^{\prime}$-simplex then $\Delta \times \Delta^{\prime}$ is an $\left(\varepsilon+\varepsilon^{\prime}\right)$-chain.

Let $x$ be a $k$-dimensional $\varepsilon$-chain of $M$ with coefficients belonging to an arbitrary abelian group $\mathfrak{A}$ and let $\varkappa^{\prime}$ be a $k^{\prime}$-dimensional $\varepsilon^{\prime}$-chain of $M^{\prime}$ with integral coefficients. Let $x=\sum_{\nu=1}^{n} \alpha_{\nu} \cdot \Delta_{\nu}$ and $x^{\prime}=\sum_{\nu^{\prime}=1}^{n^{\prime}} \alpha_{\nu^{\prime}}^{\prime} \cdot \Delta_{\nu^{\prime}}^{\prime}$ be the representations of $x$ and $x^{\prime}$ respectively consistent with the orders $\zeta$ and $\dot{\gamma}$. Putting

$$
\varkappa \times \varkappa^{\prime}=\sum_{\nu=1}^{n} \sum_{\nu=1}^{n^{\prime}} \alpha_{\nu} \cdot a_{\nu^{\prime}}^{\prime} \cdot \Delta_{\nu} \times \Delta_{\nu^{\prime}}^{\prime}
$$

we obtain a $\left(k+k^{\prime}\right)$-dimensional $\left(\varepsilon+\varepsilon^{\prime}\right)$-chain of $M \times M^{\prime}$ with the coefficients belonging to $\mathfrak{A}$, called the product of the chains $*$ and $x^{\prime}$. It is known ${ }^{2}$ ) that

$$
\partial\left(x \times x^{\prime}\right)=(-1)^{k} \cdot x \times \partial x^{\prime}+\partial x \times x^{\prime} .
$$

It follows that:

(3) If $\gamma$ and $\gamma^{\prime}$ are cycles, then $\gamma \times \gamma^{\prime}$ is a cycle.

(4) If $\gamma$ is an $\varepsilon$-cycle $\eta$-homologous to zero in $M L$ and $\gamma^{\prime}$ is an $\varepsilon^{\prime}$-cycle in $M^{\prime}$, then $\gamma \times \gamma^{\prime}$ is an $\left(\varepsilon+\varepsilon^{\prime}\right)$-cycle $\left(\eta+\varepsilon^{\prime}\right)$-homologous to zero in $M \times M^{\prime}$.

4. True chains. A sequence of chains $\underline{\dot{x}}=\left\{x_{n}\right\}$ is called a true $k$-dimensional chain of $M$ if there exists a compact subset $M_{0}$ of $M$ and a sequence $\left\{\varepsilon_{n}\right\}$ of positive numbers convergent to zero and such that $\varkappa_{n}$ is a $k$-dimensional $\varepsilon_{n}$-chain of $M_{0}$ (the coefficients of $\varkappa_{n}$ belong to an abelian group $\mathfrak{A}_{n}$, in general depending on $n$ ). If we multiply each of the chains $\varkappa_{n}$, constituting a true chain $\underline{x}$, by an integer $m$ then we obtain a true chain, which we denote by $m \cdot \underline{x}$.

If $\underline{x}=\left\{x_{n}\right\}$ and $\underline{\lambda}=\left\{\lambda_{n}\right\}$ are two $k_{\text {-dimensional true chains }}$ of $M$ and for every $n=1,2, \ldots$ the coefficients of $x_{n}$ and $\lambda_{n}$ belong to the same abelian group $\mathfrak{A}_{n}$, then the sequence $\left\{x_{n}+\lambda_{n}\right\}$ is a $k$-dimensional true chain of $M$ called the sum $\underline{x}+\underline{\lambda}$ of the chains $\underline{\varkappa}$ and $\underline{\lambda}$.

The true chain $\gamma=\left\{\gamma_{n}\right\}$ of the space $M$ such that every one of the chains $\gamma_{n}$ is a $k$-dimensional cycle is called a $k$-dimensional true cycle of $M$. If $\underline{\varkappa}=\left\{\varkappa_{n}\right\}$ is a true $(k+1)$-dimensional chain of $\boldsymbol{M}$, 
then the sequence $\left\{\partial \%_{n}\right\}$ is a true $k$-dimensional cycle of $M$, called the boundary $\partial \underline{x}$ of the true chain $\underline{\varkappa}$. A true $k$-dimensional cycle $\gamma$ of $M$ is said to be homologous to zero in $M$ (notation: $\gamma \sim 0$ in $M$ if there exists a true $(k+1)$-dimensional chain $\underline{x}$ of $\frac{\gamma}{M}$ such that $\partial \underline{\alpha}=\underline{\gamma}$. We say that a true cycle $\underline{\gamma}$ is weally homologous to zero in $M$, if there exists an integer $n_{0} \neq \overline{0}$. such that the true cycle $n_{0} \cdot \underline{\gamma}$ is homologous to zero in $M$.

A true cycle $\gamma=\left\{\gamma_{n}\right\}$ of $M$ such that all coefficients of the cycles $\gamma_{n}$ belong to an arbitrarily given abelian group $\mathfrak{P}$ is said to be convergent in $M$ if the true cycle $\left\{\gamma_{n+1}-\gamma_{n}\right\}$ is homologous to zero in $\boldsymbol{M}$.

All $k$-dimensional true cycles with rational coefficients convergent in $M$ constitute a group $C^{k}(M)$ and the k-dimensional true cycles with rational coefficients convergent and homologous to zero in $M$ constitute its subgroup $H^{k}(M)$. The rank of the factor group $C^{k}(M) / H^{k}(M)$ is said to be the $k$-th Betti number of $M$. We denote it by $p^{k}(M)$.

Let $M$ and $M^{\prime}$ be two metric spaces and let $\zeta$ denote an ordering relation in $M$ and $\left\{\right.$ an ordering relation in $M^{\prime}$. If $x=-\left\{x_{n}\right\}$ is an arbitrary $k$-dimensional true chain in $M$ and $\underline{x}^{\prime}=\left\{x_{n}^{\prime}\right\}$ a $k^{\prime}$-dimensional true chain in $M^{\prime}$ with integral coefficients then putiting

$$
\underline{x} \times \underline{x}^{\prime}=\left\{x_{n} \times x_{n}^{\prime}\right\}
$$
we obtain a $\left(k+k^{\prime}\right)$-dimensional true chain in $M \times M^{\prime}$. With regard
to (2) we have

$$
\partial\left(\underline{x} \times \underline{x}^{\prime}\right)=(-1)^{k} \underline{x} \times \partial \underline{x}^{\prime}+\partial \underline{x} \times \underline{x}^{\prime} .
$$

It follows that if $\underline{\gamma}$ and $\underline{\gamma}^{\prime}$ are true cycles then $\gamma \times \gamma^{\prime}$ is also a true cycle and if $\underline{\gamma}$ (or $\underline{\gamma}^{\prime}$ ) is homologous (or weakly homologons) to zero in $M$ then $\underline{\gamma} \times{\underline{\gamma^{\prime}}}^{\text {is homologous (or weakly homologous) }}$ to zero in $M \times M^{\prime}$. And if the true cycles $\gamma$ and $\gamma^{\prime}$ are convergent then also the true cycle $\underline{\gamma} \times \underline{\gamma^{\prime}}$ is convergent.

5. Mappings of true chains. Let $f$ be a mapping of a metric space $M$ in a metric space $M^{\prime}$. If we assign to each simplex $\Delta=\left(a_{0}, a_{1}, \ldots, a_{k}\right)$ of $M$ the simplex $\Delta_{f}=\left(f\left(a_{0}\right), f\left(a_{1}\right), \ldots, f\left(a_{k}\right)\right)$ of $M^{\prime}$ then we obtain a transformation mapping each $k$-dimensional chain $\varkappa$ of $\boldsymbol{M}$ into a $k$-dimensional chain $x_{f}^{\prime}$ of $M^{\prime}$. Evidently this chainmapping commutes with addition and with the operation of
If the mapping $f$ is continuous then we see at once that the corresponding chain mapping assigns to every $k$-dimensional true

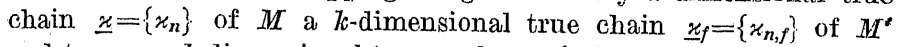
and to every $k$-dimensional true cycle $\gamma=\left\{\gamma_{n}\right\}$ of $M$ a $k$-dimensional true cycle $\underline{\gamma}_{f}=\left\{\gamma_{n, f}\right\}$ of $M^{\prime}$ and it commutes with addition and with the operation of boundary $\partial$. Moreover if the true cycle $\gamma=\left\{\gamma_{n}\right\}$ is convergent in $M$, then the corresponding true cycle $\gamma_{f}=\left\{\gamma_{n, t}\right\}$ is convergent in $M^{\prime}$.

If $f$ is a homeomorphism mapping $M$ on $M^{\prime}$ then we obtain in this manner a (1-1)-correspondence between the true cycles of the spaces $M I$ and $M I^{\prime}$ conserving the convergence and the relation of homology.

6. Cycles on $S_{k}$. The $k$-dimensional sphere $S_{k}$ is homeomorphic with the boundary $B_{k}$ of a $(k+1)$-dimensional simplex $\Delta_{k+1}$. Consequently instead of the cycles on $S_{k}$ we shall consider the cycles on $B_{k}$. Let $P_{k}$ denote the complex made up of all $k$-dimensional faces of $\Delta_{k+1}$. Choosing a positive orientation in $P_{k}$, let us denote by $\pi_{k, n}$ the $k$-dimensional cycle defined as the sum of all $k$-dimensional positively orienter simplexes of the $n$-th barycentric subdivision of $P_{k}$. It is known that the sequence $\underline{\pi}_{k}=\left\{\pi_{k, n}\right\}$ is a convergent $k$-dimensional true cycle of the polytope $\bar{B}_{k}$ and that, for each $k$-dimensional true cycle $\underline{\gamma}=\left\{\gamma_{n}\right\}$ of $B_{k}$ with the coefficients of $\gamma_{n}$ belonging to a group $\mathfrak{N}_{n}$, there exists a sequence $\left\{a_{n}\right\}$ such that. $\alpha_{n} \in \mathfrak{A}_{n}$ and that $\underline{\gamma}$ is homologous in $B_{k}$ to the true cycle $\left\{\alpha_{n} \cdot \pi_{k, n}\right\}$. Evidently the last cycle is homologous to zero in $B_{k}$ if and only if almost all coefficients $\alpha_{n}$ vanish.

Since $S_{k}$ and $B_{k}$ are homeomorphic, we infer that:

There caists in $S_{k}$ a convergent $k$-dimensional true cycle $\underline{g}=\left\{\sigma_{k, n}\right\}$ with integral coefficients such that for every $k$-dimensional true eycle $\underline{\gamma}=\left\{\gamma^{\prime} n\right\}$ of $S_{k}$, with the coefficients of $\gamma_{n}$ belonging to an abelian group $\mathfrak{Q}_{n}$, there exists a sequence $\left\{\alpha_{n}\right\}$ with $\alpha_{n} \in \mathfrak{R}_{n}$ such that $\gamma$ is homologons in $S_{k}$ to the true cycle $\left\{a_{n} \cdot \sigma_{k, n}\right\}$. The last cyale is homologous to $z$ cro in $S_{k}$ if and only if almost all coefficients $a_{n}$ vanish.

\section{Spherical cycles and spherically essential cycles.} A $k$-dimensional true cycle $\gamma$ of a metric space $M$ will be said to be spherical if there exists a $k$-dimensional true cycle $\underline{y}^{\prime}$ in $S_{k}$ and a continuous mapping $f \in M^{s_{k}}$ such that

$$
\underline{\gamma} \sim \underline{\gamma}_{f}^{\prime} \text { in } M \text {. }
$$


A $k$-dimensional true cycle $\gamma$ of $M$ will be called spherically essential in $M$ if there exists a continuous mapping $f$ of $M$ in $S_{k}$ such that $\gamma_{f}$ is not homologons to zero in $\mathbb{S}_{\boldsymbol{h}}$. Evidently if $\gamma$ is homologous to zero in $M$ then $\gamma$ is not spherically essential in $M$.

Theorem ${ }^{3}$ ). Let $M$ be a metrio space of dimension $\leqslant k$. Fach $k$-dimensional true cycle $y$ not homologous to zero in $M$ is spherically essential in $M$.

First we establish a lemma constituting a slight extension of the known lemma by Lebesgue 4 .

Lemma. Let $A_{1}, A_{2}, \ldots, A_{r}$ be compact subsets of a metrie space $\mathrm{E}$. For every $\eta>0$ there exists a $\delta>0$ such that if $B$ is a subset of $E$ with diameter $\leqslant \delta$ and $i_{1}, i_{2}, \ldots, i_{a}$ is a system of indices $\leqslant r$ such that $B \cdot A_{\boldsymbol{I}_{\boldsymbol{v}}} \neq 0$ for $\nu=1,2, \ldots, \alpha$, then there exists a point $a \in A_{t_{1}} \cdot A_{t_{2}} \cdot \ldots \cdot A_{i_{c c}}$ such that $\varrho(a, B)<\eta$.

Proof. Suppose that for an $\eta>0$ such a $\delta>0$ does not exist. Then for a system of indices $i_{1}, i_{2}, \ldots, i_{a}$ there exists for every natural $n$ a set $B_{n}$ with the diameter $<1 / n$ such that $B_{n} \cdot A_{i_{y}} \neq 0$ for $\nu=1,2, \ldots, \alpha$ and that

$$
\varrho\left(a, B_{n}\right) \geqslant \eta \text { for every } a \in A_{i_{1}} \cdot A_{i_{2}} \cdot \ldots \cdot A_{i_{a}} .
$$

Let $a_{v, n} \in A_{i_{v}} \cdot B_{n}$. Since $A_{l_{1}}$ is compact we can assume that the points $a_{1, n}$ converge to a point $a_{0} \in A_{i_{1}}$. Then also $a_{v, n} \rightarrow a_{0}$ for every $v=1,2, \ldots, \alpha$ and we infer that $a_{0} \in A_{i_{1}} \cdot A_{i_{2}} \cdot \ldots \cdot A_{i_{\alpha}}$. But

$$
\varrho\left(a_{0}, B_{n}\right) \leqslant \varrho\left(a_{0}, a_{1, n}\right) \rightarrow 0,
$$

hence $\varrho\left(a_{0}, B_{n}\right)<\eta$ for almost all $n$, contrary to $(6)$.

Proof of the theorem. We first show, by induction, that the theorem is true for compact spaces.

If $k=0$ then the cycles $\gamma_{n}$ constituting the 0 -dimensional true cycle $\underline{\gamma}=\left\{\gamma_{n}\right\}$ are of the form

where

$$
\gamma_{n}=\alpha_{n, 1}\left(a_{n, 1}\right)+a_{n, 2}\left(a_{n, 2}\right)+\ldots+\alpha_{n, l_{n}}\left(a_{n, l_{n}}\right)
$$

$$
a_{n, 1}+\alpha_{n, 2}+\ldots+a_{n, l_{n}}=0 .
$$

$\left.{ }^{3}\right)$ Compare P. Alexandroff, Dimensionstheorie. Ein Beitrag zur Geometrio der abgeschlossenen Mengen, Math. Ann. 106 (1.932), p. 223, where an analogous theorem is proved by another method under a slightly more restrictive hypo-
thesis.

1) See, for instance, P. Alexandroff and H. Hopf, Topologie $I$, Ber-
Since $\gamma$ is not homologous to zero in $M$ there exists an $\varepsilon>0$ and an increasing sequence of indices $\left\{n_{\nu}\right\}$ such that no one of the cycles $\gamma_{n_{\nu}}$ is $\varepsilon$-homologous to zero in $M$. It follows that the compact space $M$ is the sum of two closed sets $X_{v}$ and $Y_{v}$ such that:

$$
\text { If } x \in X_{\nu} \text { and } y \in Y_{\nu} \text { then } \varrho(x, y) \geqslant \varepsilon \text {. }
$$

(8) The sum $\beta_{\nu}$ of the coefficients $\alpha_{n_{v}, j}$ such that $a_{n_{v}, j} \in X_{\nu}$ does not vanish.

Since $M$ is compact there exists a subsequence $\left\{X_{\nu_{i}}\right\}$ of the sequence $\left\{X_{\nu}\right\}$ convergent to a set $X_{0} \subset M$. From (7) it follows that:

$$
\text { Putting }
$$

$$
X_{v_{i}}=X_{0} \text { for almost all indices } i \text {. }
$$

we have

$$
Y_{0}=M-X_{0}
$$

Hence

$$
Y_{\boldsymbol{v}_{i}}=Y_{0} \text { for almost all indices } i \text {. }
$$

110) $\quad M=X_{0}+Y_{0}$ and if $x \in X_{0}$ and $y \in Y_{0}$ then $\varrho(x, y) \geqslant \varepsilon$.

Let $\beta_{n}^{0}$ denote the sum of all coefficients $\alpha_{n, j}$ such that $a_{n, j} \in X_{0}$. By (8) and (9)

(11) $\quad \beta_{n_{v_{i}}}^{0}=\beta_{\boldsymbol{v}_{i}} \neq 0$ for almost all indices $i$.

The sphere $S_{0}$ contains only two points $p$ and $q$. Putting

$$
\begin{array}{lll}
f(x)=p & \text { for } & x \in X_{0}, \\
f(x)=q & \text { for } & x \in Y_{0},
\end{array}
$$

we obtain a continuous mapping of $M$ in $S_{0}$. The function $f$ maps the true cycle $\underline{\gamma}=\left\{\gamma_{n}\right\}$ onto the true cycle $\left\{\beta_{n} \cdot(p)-\beta_{n} \cdot(q)\right\}$ which, by (11), is not homologous to zero in $S_{0}$.

Assume now that $k>0$ and that the theorem is true (for compact spaces) for dimensions $<k$. Let $\gamma=\left\{\gamma_{n}\right\}$ be a $k$-dimensional true cycle not homologous to zero in a compact space $M$ of dimension $\leqslant k$. Then there exists a positive number $\varepsilon$ and an increasing sequence of indices $\left\{n_{v}\right\}$ such that $\gamma_{n_{v}}$ is not $\varepsilon$-homologous to 0 in $M$. Since $M$ is compact and $\operatorname{dim} M \leqslant k$, there exist open sets $V_{1}, V_{2}, \ldots, V_{r}$ covering $M$ such that for every $i=1,2, \ldots, r$ the diameter of $V_{i}$ is $<\varepsilon$ and that

$$
\operatorname{dim}\left(\bar{V}_{i}-V\right) \leqslant k-1
$$


Putting

$$
I_{0}=\sum_{i=1}^{r}\left(\bar{V}_{i}-V_{i}\right) ; \quad G_{i}=V_{i}-\sum_{i} \bar{V}_{i}
$$

we see at once that $M r_{0}$ is a closed subset of $M$ such that

$$
\operatorname{dim} M I_{0}<k
$$

and $G_{1}, G_{2}, \ldots, G_{r}$ are disjoint open subsets of $M$ with diameters $<\varepsilon$ and such that

$$
M-M_{0}=\sum_{i=1}^{r} Q_{i} \text { and } \quad M=\sum_{i=1}^{r} \bar{G}_{i}
$$

Let $\delta_{n} / 2$ denote the maximum diameter of simplexes of $\gamma_{n}$. Then $\delta_{n} \rightarrow 0$. By the lemma there exists for almost every $n$ a positive number $\eta_{n}$ such that

$$
\lim _{n=\infty} \eta_{n}=0
$$

and that for every subset $B$ of $M$ with diameter $\leqslant \delta_{n}$ if $B \cdot \bar{G}_{i_{\nu}}=0$ for $\nu=1,2, \ldots, \alpha$, then there exists a point $a \in \bar{G}_{i_{1}} \cdot \bar{G}_{i_{2}} \cdot \ldots \cdot \bar{G}_{i_{c}}$ such that $\varrho(a, B)<\eta_{n}$.

Now we associate with each vertex $p$ of $\gamma_{n}$ a point $\omega_{n}(p) \in M$ in the following manner:

Denote by $R(p)$ the set composed by all points $g \in M$ such that there exists in $\gamma_{n}$ a simplex $\Delta$ containing $p$ and $q$ among its vertices. Evidently $p \in B(p)$ and the diameter of $B(p)$ is $\leqslant \delta_{n}$.

Let $i_{1}, i_{2}, \ldots, i_{c e}$ be the maximal system of indices $\leqslant r$ such that $B(p) \cdot \bar{G}_{i_{\nu}} \neq 0$ for every $\nu=1,2, \ldots, a$. By the lemma there exists a point $a \in \bar{G}_{i_{1}} \cdot \bar{G}_{i_{2}} \cdot \ldots \cdot \bar{G}_{i_{\alpha}}$ such that $\varrho\left(a, B_{n}\right) \leqslant \eta_{n}$. We put $\omega_{n}(p)=a$.

Evidently the cycle $\omega_{n}\left(\gamma_{n}\right)$ is $\left(\eta_{n}+\delta_{n}\right)$-homologous in $M$ to the eycle $\gamma_{n}$. It follows that the cycles $\omega_{n}\left(\gamma_{n}\right)$ constitute a true $k$-dimensional cycle in $M$ and that for almost all $v$ the cycle $\omega_{n_{\nu}}\left(\gamma_{n_{\nu}}\right)$ is not $\varepsilon$-homologous to 0 in $M$.

Moreover let us observe that for almost all $n$ each simplex $\omega_{n}(\Delta)$ of $\omega_{n}\left(\gamma_{n}\right)$ having a vertex belonging to $G_{l}$ lies in $\bar{G}_{l}$. In fact, by the construction of the function $\omega_{n}$, if a vertex $\omega_{n}(p)$ of $\omega_{n}(\Delta)$ belongs to $G_{i}$ then also $p \in G_{i}$ and for every vertex $q$ of $\Delta$ it is $\omega_{n}(q) \in \bar{G}_{i}$.

Let us denote by $x_{n, i}$ the chain made up of all simplexes of $\omega_{n}\left(\gamma_{n}\right)$ having a vertex belonging to $G_{i}$ with the same coefficients as in $\omega_{n}\left(\gamma_{n}\right)$. Then

$$
\omega_{n}\left(\gamma_{n}\right)=\varkappa_{n, 0}+\varkappa_{n, 1}+\ldots+\varkappa_{n, r}
$$

where $\varkappa_{n, 0}$ is a chain lying in $M_{0}$ and for $i=1,2, \ldots, r, \varkappa_{n, i}$ is a chain lying in $\bar{G}_{i}$. Then $\partial \varkappa_{n, i}$ is, for $i=1,2, \ldots, r$, a $(k-1)$-dimensional cycle lying in the boundary $D_{t} \subset M_{0}$ of the set $G_{i}$ and $\left\{\partial \varkappa_{n, i}\right\}$ is a true (k-1)-dimensional cycle of $M_{0}$. If for every $i=1,2, \ldots, r$, the true cycle $\left\{x_{n, t}\right\}$ were homologous to zero in $D_{t}$, then there would exist a true $k$-dimensional chain $\left\{\lambda_{n, i}\right\}$ of $D_{i}$ such that $\partial \lambda_{n, i}=\partial x_{n, i}$ for every $n=1,2, \ldots, i=1,2, \ldots, r$. Since the diameter of $D_{t}$ is $<\varepsilon$ the cycle $\lambda_{n, t}-\varkappa_{n, l}$ is $\varepsilon$-homologous to zero in $\boldsymbol{M}$. It follows that the chain

$$
\varkappa_{n_{\nu}, 0}+\lambda_{n_{\nu}, 1}+\ldots+\lambda_{n_{\nu}, r}=\omega_{n_{\nu}}\left(\gamma_{n_{\nu}}\right)-\left(\varkappa_{n_{\nu}, 1}-\lambda_{n_{\nu}, 1}\right)-\ldots-\left(\varkappa_{n_{\nu}, r}-\lambda_{n_{\nu}, r}\right)
$$

would be a $k$-dimensional cycle lying in $M_{0}$ and $\varepsilon$-homologous in $M$ to $\omega_{n_{y}}\left(\gamma_{n_{y}}\right)$. But $\omega_{n_{y}}\left(\gamma_{n_{y}}\right)$ is not $\varepsilon$-homologous to zero in $M$. Hence the cycle $\varkappa_{n_{y}, 0}+\lambda_{n_{\nu}, 1}+\ldots+\lambda_{n_{\nu}, r}$ would not be $\varepsilon$-homologous to zero in $M_{0}$ and the true $k$-dimensional cycle $\left\{\kappa_{n, 0}+\lambda_{n, 1}+\ldots+\lambda_{n, \boldsymbol{r}}\right\}$ of $M_{0}$ would not be homologous to zero in $M_{0}$. But this contradicts the assumption $\operatorname{dim} M_{0} \leqslant k-1$.

It follows that there exists an index $i_{0}$ such that the true (k-1)-dimensional cycle $\delta=\left\{\partial x_{n, l_{0}}\right\}$ is not homologous to zero in $D_{i_{0}}$. By the hypothesis of the induction there exists a continuous function $\varphi$ mapping $D_{t_{0}}$ in $S_{k-1}$ and carrying the true cycle $\underline{\delta}$ into a true cycle homologous on $S_{k-1}$ to a true cycle of the form $\left\{\alpha_{n} \cdot \sigma_{k-1, n}\right\}$, where $\alpha_{n} \neq 0$ for an infinite collection of the indices $n$. We may assume that $S_{k-1}$ is the "equator" of the sphere $S_{k}$ dividing it into two halfspheres $H_{1}$ and $H_{2}$. Evidently there exists a continuous extension $f$ of $\varphi$ over $M$ such that $f\left(G_{i_{0}}\right) \subset H_{1}$ and $f\left(M-G_{i_{0}}\right) \subset H_{2}$. One readily sees that $f$ maps the true cycle $\left\{\omega_{n}\left(\gamma_{n}\right)\right\}$, hence also the true cycle $\gamma$ into the cycle homologous in $S_{k}$ to the true cycle of the form $\left\{\alpha_{n} \cdot \sigma_{k, n}\right\}$. Hence $\gamma$ is spherically essential. in $M$ and the proof of the theorem for compact spaces is complete.

Passing to the case in which $M$ is an arbitrary metric space we find a compact subset $N$ of $M$ containing $\gamma$ and a continuous function $g$ mapping $N$ in $S_{k}$ in such a manner that $\gamma_{g}$ is a true cycle not homologous to zero in $S_{k}$. Since $\operatorname{dim} M \leqslant \bar{k}$ there exists ${ }^{5}$ ) a continuous extension $f$ of $g$ over $M$ with the values lying on $S_{k}$. Then $f$ carries $\underline{\gamma}$ into the true cycle $\underline{\gamma}_{f}=\underline{\gamma}_{g}$ not homologous to zero in $S_{k}$. This proves the theorem.

5) See, for instance, W. Hurewicz and H. Wallman, l. c., p. 83. Fundamenta Aathematicac. T. XXXIX. 
8. Homology and extension of mappings. We now prove the following

Lemma. Let $f$ be a continuous mappiny of a compact space $X$. in a metrie space $Y$ and let $\gamma=\left\{\gamma_{n}\right\}$ be a true cycle lying in $X$. The true cycle $\underline{\gamma}_{f}=\left\{\gamma_{n f f}\right\}$ is homologous to zero in $Y$ if and only if there exists a continuous extension $f^{\prime}$ of $f$, with ihe values belonging to $\mathrm{V}$, over a compact spase $X^{\prime} \supset X$ such that $\underline{\gamma} \sim 0$ in $X^{\prime}$.

Proof. The condition is sufficient because the relation $\gamma \sim 0$ : in $X^{\prime}$ implies the relation $\underline{\gamma}_{f}^{\prime}=\underline{\gamma_{f}} \sim 0$ in $X$. Thus it remains to prove its necessity.

First let us observe that if $h$ is a homeomorphic mapping of $X$ onto any compact space $X_{0}$ and $\gamma_{f} \sim 0$ in $Y$ then it sufficess to show that there exists a continuous extension $\varphi$ of the mapping $f h^{-1} \in Y^{X_{0}}$ with the values belonging to $Y$, over a compact space $X_{0}^{\prime} \supset X_{0}$ such that the true cycle $\underline{\gamma}_{h}$ is homologous to zero in $X_{0}^{\prime}$.

Consequently we may assume that $X$ is a subset of the Fillbert. cube $Q_{\omega}^{6}{ }^{6}$. By our hypothesis there exists a compact set $Y_{0} \subset X$ such that $f(X) \subset Y_{0}$ and the true eycle $y_{f}$ is homologous to zero in $Y_{u}$. Let $X_{0}$ be the subset of the compact space $Q_{0} \times Y_{0}$ composed of all points of the form $x \times f(x)$ with $x \in \bar{X}$. Putting

$$
h(x)=x \times f(x) \text { for every } x \in X
$$

we obtain a homeomorphic mapping of $X$ onto $X_{1,}$. From the remark just made, we infer that it suffices to show that there exists a continuous extension $g$ of $f h^{-1}$ over a compact space $X_{0}^{\prime} \supset X_{0}$ such that the values of $g$ belong to $Y$ and that $\gamma_{h} \sim 0$ in $X_{0}^{\prime}$.

Let us put

$$
X_{0}^{\prime}=E_{p}[p=t x \times f(x), x \leqslant X \text { and } 0 \leqslant t \leqslant 1]+(0) \times Y_{0} .
$$

Evidently $X_{0}^{\prime}$ is a compact space and $X_{0} \subset X_{0}^{\prime}$. Putting

$$
\begin{array}{ll}
g(t \cdot x \times f(x))=f(x) & \text { for } x \in X \text { and } 0 \leqslant t \leqslant 1, \\
g(0 \times y)=y & \text { for } y \in Y_{0}
\end{array}
$$

6) By the Hilbert aube $Q_{\omega}$ we understand the set of points $\left\{x_{n}\right\}$ in Hilbert space whose $n$-th coordinate $x_{n}$ satisfies the inequality $0 \leqslant x_{n} \leqslant 1 / n$. If $x=\left\{x_{n}\right\} \in Q_{\omega}$ and $0 \leqslant t \leqslant 1$ then $t \cdot x$ denotes the points $\left\{t \cdot x_{n}\right\} \in Q_{\omega}$. In particular we denote by 0 the point $\left\{x_{n}\right\}$ such that $x_{n}=0$ for every $n=1,2, \ldots$ Consequently $0 \cdot x=0$ for every $x \in Q_{\omega}$. By the known theorem of P. Urysohn eacl mently $0 \cdot x=0$ space is homeomorphic to a subset of $Q_{\omega}$. we obtain a continuous function $g$ mapping $X_{0}^{\prime}$ into $Y$. The value of $g$ at the point $h(x)=x \times f(x)$ is equal to $f(x)$. Hence $g$ is an extension of $f h^{-1}$.

It remains only to show that the true cycle $\underline{\gamma}_{h}$ is homologous to zero in $X_{0}^{\prime}$. To do it let us observe that putting

$$
p_{\lambda}(x \times f(x))=\lambda \cdot x \times f(x) \text { for } x \in X \text { and } 0 \leqslant \lambda \leqslant 1
$$

we obtain a continuous family of the functions $\left\{\varphi_{\lambda}\right\}$ deforming homotopically the set $X_{0}$ in the space $X_{0}^{\prime}$. By this homotopic deformation the true cycle $\underline{\gamma}_{h}=\underline{\gamma}_{h \varphi_{1}}$ is carried into the true cycle $\gamma_{h \varphi_{0}}$ being the image of the true cycle $\gamma_{f}$ by the homeomorphic mapping $y \rightarrow 0 \times y$ of the set $\bar{Y}_{0}$ on the set $(0) \times \bar{Y}_{0}$. But the true cycle $y^{\prime}$ is homologous to zero in $Y_{0}$, hence $\underline{\gamma}_{h \varphi_{0}}$ is homologous to zero in $(0) \times Y_{0} \subset X_{0}^{\prime}$. Thus we have

$$
\underline{\gamma}_{h} \sim \underline{\gamma}_{h \varphi_{0}} \text { in } X_{0}^{2} \text { and } \underline{\gamma}_{h \varphi_{0}} \sim 0 \quad \text { in } X_{0}^{\prime}
$$

and finally $\underline{\gamma}_{h} \sim 0$ in $X_{0}^{\prime}$. This proves our lemma.

9. Cycles in the functional space. We now come to the main result of this paper.

Theorem. If a compact space $X$ contains a k-dimensional spherically essential true cycle $\underline{\gamma}=\left\{\gamma_{n}\right\}$, with arbitrary coefficients, then the functional space $\aleph_{m}^{X}(m \geqslant k)$ contains a convergent $(m-k)$-dimensional true spherical cycle $\underline{\underline{\gamma}}^{*}$ with integral coefficients not homologous to zero in $S_{m}^{X}$.

In the case for which almost all of the groups $\mathfrak{U}_{n}$, to which the coefficients of $\gamma_{n}$ belong, contain no elements of finite order, the true cycle $\underline{\gamma}^{*}$ is not weakly homologous to zero in $S_{m}^{X}$.

Proof. By Nrs 6 and 7 there exists a mapping $\varphi \in S_{k}^{X}$ such that the true cycle $\gamma_{\varphi}$ is homologous in $S_{k}$ to a true cycle of the form $\left\{a_{n} \cdot \sigma_{k, n}\right\}$ where $a_{n} \in \mathfrak{A}_{n}$ and $a_{n} \neq 0$ for an infinite set of indices $n$. Putting

$$
f(x \times y)=p(x) \times y \text { for } \quad x \times y^{\prime} \epsilon X \times S_{m-k}^{\prime},
$$

we obtain a continuous mapping $f$ of the product $X \times \aleph_{m-k}$ onto the $m$-dimensional orientable manifold $S_{k} \times S_{m-k}$ such that the true $m$-dimensional cycle $\left\{\gamma_{n} \times \sigma_{m-k, n}\right\}$ is carried by $f$ into an $m$-dimensional true cycle homologous in $S_{k} \times S_{m-k}$ to the true cycle $\left\{\alpha_{n} \cdot \sigma_{k, n} \times \sigma_{m-k, n}\right\}$. 
Now let us consider a continuous mapping. $\psi$ of the m-dimensional orientable manifold $S_{k} \times S_{m-k}$ onto the sphere $S_{m}$ carrying, the true $m$-dimensional convergent cycle $\left\{\sigma_{k, n} \times \sigma_{m-k, n}\right\}$ with integral coefficients into a convergent true cycle homologous in $S_{m}$ to the cycle $\left\{\sigma_{m, n}\right\}$. We readilly see that $\psi f$ maps the product $X \times s_{m-k}$ into $S_{m}$ in such a manner that it carries the true cycle $\left\{\gamma_{n} \times \sigma_{m-k, n}\right\}$ into a true cycle homologous in $S_{m}$ to the true cycle $\left\{\alpha_{n} \cdot \sigma_{m, n}\right\}$. If we assign to each point $y \in S_{m-k}$ the mapping $g_{y} \in S_{m}^{X}$ defined by the formula

$$
g_{y}(x)=\psi f(x \times y)
$$

then we obtain a continuous mapping $g$ of the sphere $s_{m-k}$ into the space $S_{m}^{X}$. The mapping $g$ carries the convergent true $(m-k)$-dimensional cycle $\left\{\sigma_{m-k, n}\right\}$ into a convergent true $(m-k)$-dimensional spherical cycle $\underline{\gamma}^{*}$ lying in $S_{m}^{X}$ and having integral coefficients.

Were $\gamma^{*}$ homologous to zero in $S_{m}^{X}$ then, by the lemma of Nr 8, there would exist a compact space $Q \supset S_{m-k}$ such that the true cycle $\left\{\sigma_{m-k, n}\right\}$ is homologous to zero in $Q$, and a continuous extension $g^{\prime}$ of $g$ over $Q$, with the values belonging to $S_{m}^{X}$. The mapping $g^{\prime}$, being an extension of $g$, carries the true cycle $\left\{\sigma_{m-k, n}\right\}$ into $\gamma^{*}$ and it assigns to each point $y \in Q$ a mapping $g^{\prime}(y)=q_{y} \in S_{m}^{X}$. Putting

$$
\vartheta(x \times y)=\varphi_{y}(x) \text { for } x \times y \in X \times Q
$$

we obtain a continuous function $\vartheta$ mapping $X \times Q$ into $S_{m}$. The mapping $\vartheta$ coincides in the set $X \times S_{m-k} \subset X \times Q$ with the mapping $\psi f$ and consequently it maps the $m$-dimensional true cycle $\left\{\gamma_{n} \times \sigma_{m-k, n}\right\}$ onto a true cycle homologous to $\left\{\alpha_{n} \cdot \sigma_{m, n}\right\}$ in $S_{m}$.

But the relation

implies

$$
\left\{\sigma_{m-k, n}\right\} \sim 0 \text { in } Q
$$

$$
\left\{\gamma_{n} \times \sigma_{m-k, n}\right\} \sim 0 \text { in } X \times Q .
$$

It follows that $\vartheta$ maps $\left\{\gamma_{n} \times \sigma_{m-k, n}\right\}$ into a true cycle homologous to zero in $S_{m}$. Hence

$$
\left\{a_{n} \cdot \sigma_{m, n}\right\} \sim 0 \text { in } S_{m}
$$

and finally $a_{n}=0$ for almost all indices $n$, contrary to our assumption.

Let us assume now that almost all of the groups $\mathfrak{A}_{n}$ contain no elements of finite order. If $\underline{\gamma}^{*}$ were weakly homologous to zero in $S_{m}^{X}$ then there would exist an integer $n_{0} \neq 0$ such that

$$
n_{0} \cdot \underline{\gamma}^{*} \sim 0 \text { in } S_{m}^{X}
$$

Repeating the reasoning just applied we infer that there would exist a compact set $Q \supset S_{m-k}$ such that

$$
\left\{n_{0} \cdot \sigma_{m-k, n}\right\} \sim 0 \text { in } Q
$$

and a continuous function $\vartheta$ mapping $X \times Q$ into $S_{m}$ in such a manner that it carries the $m$-dimensional true cycle. $\left\{\gamma_{n} \times n_{0} \cdot \sigma_{m-k, n}\right\}$ into a true cycle homologous to $\left\{n_{0} \cdot a_{n} \cdot \sigma_{m, n}\right\}$ in $S_{m}$. It follows that

$$
\left\{n_{0} \cdot \alpha_{n} \cdot \sigma_{m, n}\right\} \sim 0 \text { in } S_{m}
$$

and consequently $n_{0} \cdot a_{n}=0$, hence also $\alpha_{n}=0$ for almost all indices $n$. But this contradicts our assiumption.

Combining the last theorem with the theorem of $\mathrm{Nr} 7$ we obtain the following

Corollary. If $X$ is a compact space such that $\operatorname{dim} X \leqslant k$ and $p^{k}(X)>0$ then $p^{m-k}\left(S_{m}^{X}\right)>0$ for $m=k, k+1, \ldots$

Problem 1. Let $X$ be a compact space of dimension $\leqslant k$ and such that every true $k$-dimensional cycle of $X$ is homologous to zero in $X$. Is it true that $p^{m-k}\left(S_{m}^{X}\right)=0$ for $m=k . k+1, \ldots$ ?

Problem 2. Let $X$ be a compactum of dimension $\leqslant k$. Do the homological properties of the space $S_{m}^{X}$ (for $\left.m=k, k+1, \ldots\right)$ depend only on the homological properties of $X$ ?

Problem 3. Is the space $\mathrm{s}_{2}^{S_{1}}$ unicoherent?

Państwowy Instytut Matematyczny. 